\title{
Expression of the melanoma-associated antigen is associated with progression of human thyroid cancer
}

\author{
Sonia Cheng ${ }^{1,2}$, Wei Liu' ${ }^{1}$, Moises Mercado ${ }^{2}$, Shereen Ezzat ${ }^{3}$ \\ and Sylvia L Asa $^{\top}$
}

\author{
${ }^{1}$ Department of Pathology, University Health Network and the Ontario Cancer Institute, 200 Elizabeth Street, 11th Floor, Toronto, \\ Ontario, Canada M5G 2C4 \\ ${ }^{2}$ Servicio de Endocrinología, Unidad de Investigación en Endocrinología Experimental, Hospital de Especialidades, IMSS, Mexico \\ City, Mexico \\ ${ }^{3}$ Department of Medicine, University Health Network and the Ontario Cancer Institute, 600 University Avenue, Toronto Ontario, \\ Canada M5G 1X5
}

(Correspondence should be addressed to S L Asa is at Department of Pathology, University Health Network and the Ontario Cancer Institute; Email: sylvia.asa@uhn.on.ca)

\begin{abstract}
Thyroid cancer exhibits a spectrum from relatively indolent tumors to tumors that are invasive, metastatic, or progress to poorly differentiated carcinoma. Microarray expression analysis of thyroid cancer cell lines has implicated a member of the melanoma-associated (MAGE) family of cancer-testis antigens in thyroid cancer development and progression. We performed this study to validate the role of MAGE in human thyroid cancers. A tissue microarray (TMA) of samples from 375 patients with thyroid cancer was analyzed with immunohistochemistry (IHC) to localize MAGE. Western blotting of fractionated proteins from MAGE-transfected cells was used to confirm intracellular localization of proteins. Automated analysis of TMA samples was evaluated and subjected to statistical analysis. MAGE immunoreactivity was identified in nuclear and cytoplasmic compartments of normal and malignant tissues. Specificity of staining was proved by fractionation studies that confirmed MAGE expression in nucleus and cytoplasm. Normal thyroid tissue exhibited weak cytoplasmic and strong nuclear MAGE reactivity. Tumors exhibited an increase in cytoplasmic MAGE scores that correlated with clinical behavior: larger tumors had higher MAGE scores, and there was a positive and significant correlation between MAGE cytoplasmic score and the number of histologically proven lymph node metastases. There was a statistically significant negative correlation between cytoplasmic MAGE and the percentage of p53-positive nuclei. Our data confirm gene-profiling evidence that members of the MAGE family play a role in thyroid cancer progression. The use of TMA analyses identifies IHC techniques that are translatable to the clinical setting for prognostic assessment of patients with thyroid cancer.
\end{abstract}

Endocrine-Related Cancer (2009) 16 455-466

\section{Introduction}

Thyroid cancer, the most common form of endocrine neoplasia, represents an excellent model to study cellular differentiation processes (Asa 2005). It exhibits a spectrum of behaviors from the small, well-differentiated papillary microcarcinoma of low clinical risk, to the large, rapidly invasive, metastatic, and lethal anaplastic carcinoma. The variable clinical traits and pathological findings are associated with alternative and complex molecular events (Kondo et al. 2006).
Melanoma-associated gene expression (MAGE) antigens were first described in a melanoma cell line. This is a 12-member family of proteins encoded on chromosome $\mathrm{Xq} 28$. The precise cellular expression and biological functions of the MAGE antigens have not been completely elucidated. Also referred to as 'cancer-testis antigens', they are not expressed in normal tissues except the testis and placenta. In neoplastic lesions, they have been found to be expressed in 12-60\% of solid malignant tumors including lung (Fischer et al. 
1997, Jang et al. 2001), liver (Kobayashi et al. 2000), endometrial (Chitale et al. 2005), and genitourinary carcinomas (Hudolin et al. 2006, Picard et al. 2007) and cholangiocarcinomas (Tsuneyama et al. 2004).

Recent work from our group using gene microarray analysis has identified the MAGE-A3 member as a target of the fibroblast growth factor receptor (FGFR2IIIb; Kondo et al. 2007b). Normal thyroid tissue expresses FGFR2-IIIb that has tumor-suppressive effects (St Bernard et al. 2005, Kondo et al. 2007a), including suppression of MAGE-A3 expression (Kondo et al. 2007b). By contrast, expression of FGFR2 is lost in thyroid tumors (St Bernard et al. 2005). Forced MAGEA3 expression results in deregulated cell cycle control through transcriptional downregulation of p53. Immunodeficient mice xenografted with human thyroid carcinoma cells forced to express MAGE-A3 showed more rapid tumor development and significantly enhanced pulmonary metastases. We have shown that loss of the cell adhesion factor fibronectin results in significantly enhanced tumor progression and metastatic spread in an orthotopic mouse model (Liu et al. 2007). Interestingly, gene profiling also identified MAGE-A3 as a target of fibronectin downregulation (Kondo et al. 2007b). Further studies by our group have demonstrated that fibronectin-mediated tumor progression is dependent on MAGE-A3 action (Liu et al. 2008). Also, as shown by us and others, MAGE-A3 downregulation of p53 is mediated through histone deacetylase recruitment to the p53 promoter (Monte et al. 2006).

In the present study, we examine the pattern of MAGE protein expression in human thyroid cancer progression, its correlation with nuclear $\mathrm{p} 53$ positivity and its possible impact on clinical tumor behavior.

\section{Materials and methods}

\section{Patients}

We identified 375 patients who underwent thyroid resection for thyroid cancer at the Hospital de Especialidades, IMSS, Mexico City, from 1992 to 2008 and at the University Health Network (UHN), Toronto, from 2002 to 2004 from clinical files and pathological archives respectively. The study protocol was approved by the local Institutional Review Boards. All available clinical data, surgical and pathology reports, as well as available follow-up were recorded.

\section{Thyroid specimens}

Paraffin blocks of formalin-fixed tissues were collected and in each case, the most representative areas of tumor, normal surrounding tissue, invasive tumor, and metastatic tissue were selected whenever available. Tissue microarrays (TMAs) were built using the semi-automated arrayer TMArrayer (Pathology Devices, Westminster, MD, USA). Triplicate cores were obtained from every tissue type, whenever possible.

\section{Immunohistochemistry}

Four-micron-thick sections of paraffin blocks were dewaxed in five changes of xylene and rehydrated through graded alcohols into water. For antigen retrieval, sections were heat-treated inside a decloaking chamber (Biocare, Birmingham, UK) in $10 \mathrm{mM}$ citrate buffer at $\mathrm{pH} 6.0$ except for MAGE, which was retrieved with Tris-EDTA buffer at $\mathrm{pH}$ 9.0. Endogenous peroxidase and biotin activities were blocked respectively using 3\% hydrogen peroxide and an avidin/biotinblocking kit (Lab Vision, Fremont, CA, USA). Sections were treated for $15 \mathrm{~min}$ with $10 \%$ normal horse serum (Vector Labs, Burlingame, CA, USA) and then incubated with the appropriate primary antibody.

Two antibodies were applied for MAGE immunolocalization. Optimization was performed using sections from formalin-fixed, paraffin-embedded ellets of WRO thyroid carcinoma cells transfected with a MAGE-A3 expression vector or control empty vector. The MAGE-A3/A6 monoclonal antibody C-13 (Santa Cruz Biotechnology, Santa Cruz, CA, USA) yielded no specific staining, whereas the $6 \mathrm{C} 1$ mouse monoclonal antibody (Santa Cruz Biotechnology) resulted in nuclear and cytoplasmic localization that was not present in control cells and corresponded to the results of western blots of fractionated cell lysates (see below).

For TMA studies, the primary reactions were as follows: anti-MAGE (6C1) mouse monoclonal antibody (1:50; Santa Cruz Biotechnology) overnight; p21 (1:300; BD Pharmingen, San Diego, CA, USA) overnight; p53 DO7 mouse monoclonal (1:300; Novocastra, Wetzlar, Germany) for $1 \mathrm{~h}$. This incubation was followed by 30 min each with biotinylated horse anti-mouse IgG (Vector Labs) and HRPconjugated Ultra Streptavidin Labeling Reagent (ID Labs Inc., London, ON, Canada). Color development was performed with freshly prepared NovaRed solution (Vector Labs Inc.) and counterstained with Mayer's hematoxylin. Sections were dehydrated through graded alcohols, cleared in xylene, and mounted in Permount (Fisher, Waltham, MA, USA).

\section{Fractionation experiments}

Protein lysates of WRO cells stably transfected with human MAGE-A3 or empty vector as previously 
described (Liu et al. 2007) were fractionated into nuclear and cytoplasmic compartments using the Nuclei EZprep isolation Kit (Sigma). The protein lysates were blotted with specific antibodies for dimethyl-histone $\mathrm{H} 3$ as a control for the nuclear fraction (Upstate, Billerica, MA, USA), MAGE (6C1 monoclonal, Santa Cruz Biotechnology), and actin (manufacturer) according to previously described protocols.

\section{Automated analysis}

The immunohistochemistry (IHC) slides were scanned using the Aperio ScanScopeXT scanner and analyzed with the spectrum-automated analyzer (Aperio, Vista, CA, USA) with the Color Deconvolution V9.0 algorithm for cytoplasmic stain. The resulting variables were average weak cytoplasmic intensity, medium cytoplasmic intensity, and strong cytoplasmic intensity scores as referenced by the cytoplasmic levels of protein expression, percentage of positive cells, and area of analysis. The software generated a construct variable named MAGE total cytoplasmic score (0-300) obtained by a calculation of area, number of positive cells, and intensity. The nuclear protein score was derived from the nuclear algorithm, and the main variable analyzed was the percentage of positive nuclei. The results were consistent with an initial manual scoring of a subset of samples, in which two observers obtained an interobserver $\kappa$ value of 0.82 for nuclear and 0.84 for cytoplasmic staining, crossvalidating the methods of analyses.

\section{Statistical analysis}

Variables are presented as S.E.M. or median and interquartile range according to their distribution. $T$-test for comparison of means, ANOVA, and Pearson's correlation if they had a normal distribution. Non-parametric statistical analysis was used when appropriate for comparison of median values, KruskalWallis test for multiple groups, and Mann-Whitney $U$-tests for two independent groups. Statistical significance was considered reached at $P<0.05$.

\section{Results}

\section{TMA cores}

A total of 1184 cores were examined, including the triplicates. Out of these, $741(62.44 \%)$ represented primary tumors, $286(24.25 \%)$ were non-neoplastic tissue surrounding the tumor, and 157 (13.31\%) came from metastatic tumor tissue (Fig. 1A).
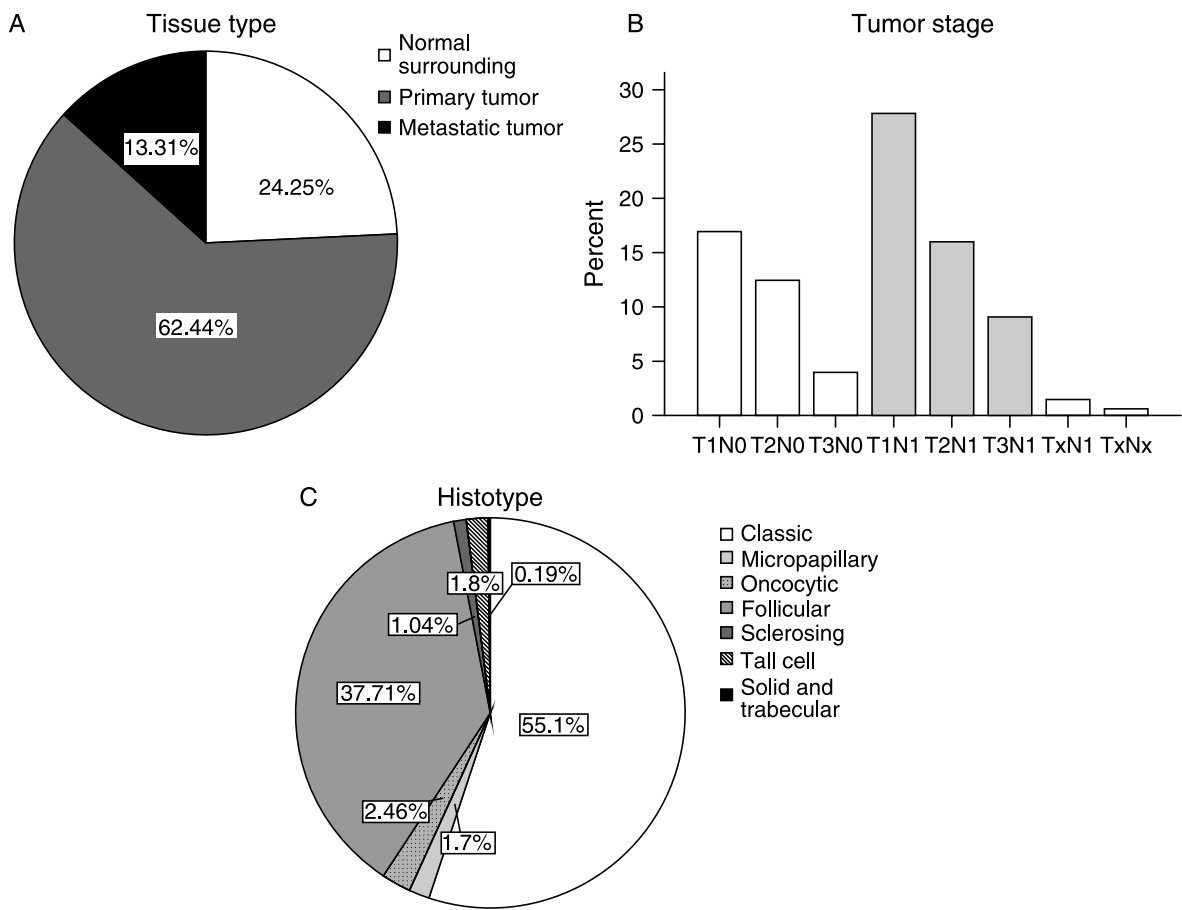

Figure 1 Distribution of tissues used in thyroid TMA. Tissues were grouped according to (A) tissue type, (B) tumor stage, and $(\mathrm{C})$ histotype. As observed in the figure, only $40 \%$ of the primary tumors have a corresponding normal surrounding tissue sample, and $22 \%$ have a metastatic sample; $45 \%$ were Stage T1, $56.8 \%$ were classified as papillary (including micropapillary), and $37.71 \%$ were follicular variant papillary carcinoma. 


\section{Clinical and pathological variables}

From the 375 patients with complete clinical and pathologic diagnoses, 305 had specific information about gender, 280 had detailed information on extrathyroidal extension, and 215 cases were evaluated for the presence of lympho-vascular invasion. The most frequent reason for surgical intervention was suspicious thyroid nodule $(78.4 \%)$, followed by multi-nodular goiter with dominant nodule (6.1\%). Only $4.3 \%$ of the cases represented incidental findings and $3.7 \%$ were thyroid nodules in previously hyperthyroid patients mainly, Graves' disease.

The mean age at diagnosis was 45.14 years $(16-88$, S.E.M. $=0.43)$, mean tumor size was $2.56 \mathrm{~cm}(0.3-14$, S.E.M. $=0.059$ ), and in cases with lymph node metastases, the mean number of positive lymph node metastases was $3.79(1-44$, S.E.M. $=0.23)$. Tumor stage, according to the 2004 AJCC classification, was $\mathrm{T} 1 \mathrm{~N} 0$ in $17.6 \%$ of the cases, T1N1 in $26.1 \%$, T2N0 in $12.8 \%$, T2N1 in $16.3 \%$, T4N0 in $5.1 \%$, and $7.5 \%$ were labeled as T4N1 (Fig. 1B). Only $1.8 \%$ remained as $\mathrm{TxN} 1$ or $\mathrm{TxN} 0$ and $12.8 \%$ were non-classifiable due to incomplete information.

As expected, $92.3 \%$ of cases represented papillary thyroid cancer, $2.7 \%$ poorly differentiated, $2.1 \%$ follicular thyroid carcinoma, $2.1 \%$ medullary thyroid carcinoma, and $0.8 \%$ anaplastic carcinoma. These proportions, in contrast to the classical pathology literature, are attributed to the recognition of subtypes of papillary carcinoma, particularly the follicular variant. The most frequent histotype within the papillary carcinomas was the classic variant $(55.1 \%)$, followed by the follicular variant $(37.7 \%)$. The oncocytic, tall-cell, sclerosing, micropapillary, solid, and trabecular variants of papillary carcinoma were each represented by $\sim 3 \%$ of cases (Fig. 1C).

The extent of local disease was evaluated; $67.9 \%$ of the tumors were confined to the thyroid and $32.1 \%$ had extra-thyroidal extension. Only $7 \%$ of tumors had unequivocal vascular invasion, whereas $93 \%$ were negative for this parameter.

\section{MAGE immunostaining}

Representative figures illustrating the results of IHC are shown in Fig. 2. MAGE positivity was identified in the nuclear and the cytoplasmic compartments. Clear differences were observed among the different tissue types. Normal thyroid tissue exhibited nuclear reactivity with only faint cytoplasmic staining. Thyroid cancers exhibited a progression of increased cytoplasmic reactivity with further increased intensity in the metastatic tumors.

Cytoplasmic MAGE immunoreactivity scores confirmed significantly higher expression in primary tumors compared with matched normal surrounding tissue $(66.17$ (S.E.M. $=1.05)$ vs 49.7 (s.E.M. $=1.62$ ), $P<0.0001$; Fig. 3A). This increase in MAGE expression was also noted in tumor metastases compared with their normal surrounding tissue (69.0 (s.E.M. $=2.3$ ) vs 49.71 (s.E.M. $=1.62), P<0.0001$ ). There was no significant difference in MAGE immunoreactivity between any given primary tumor
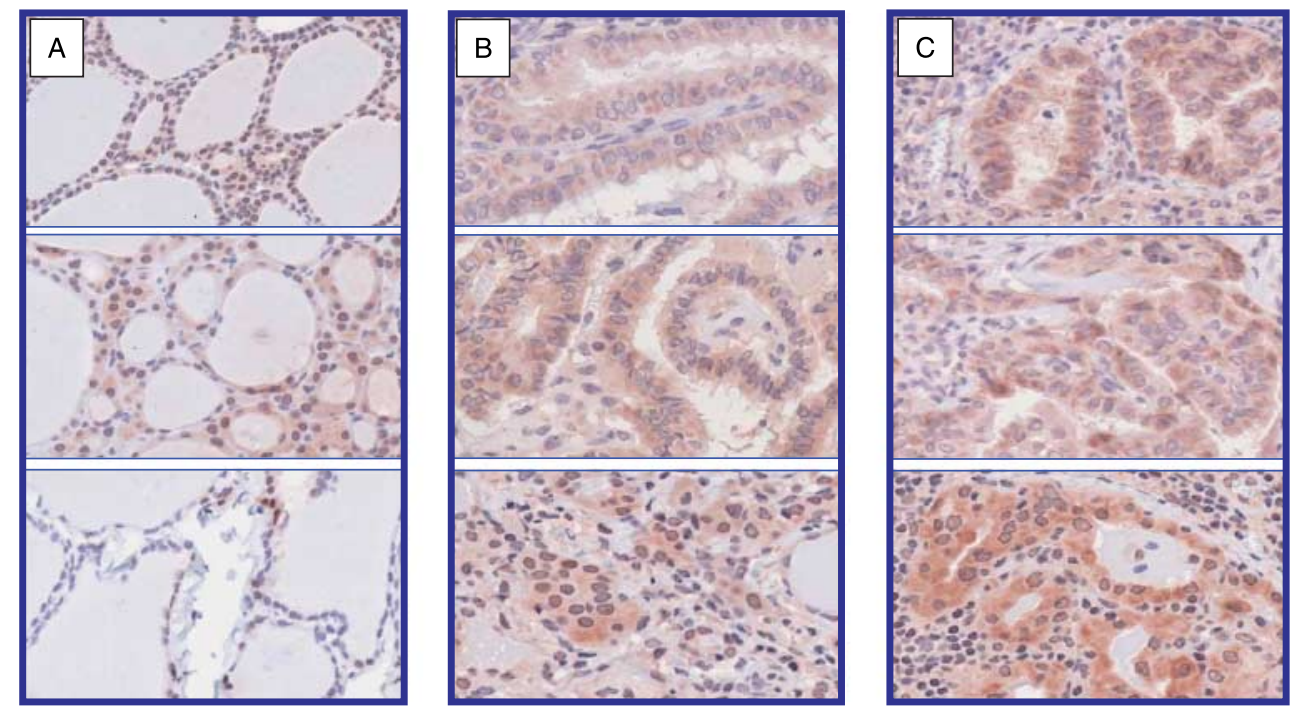

Figure 2 Immunohistochemistry for MAGE proteins. (A) Predominantly nuclear staining can be observed in the normal tissue, (B) cytoplasmic and nuclear in the primary tumor with variable intensities, and (C) a predominantly intense cytoplasmic staining in the metastatic tissues. 

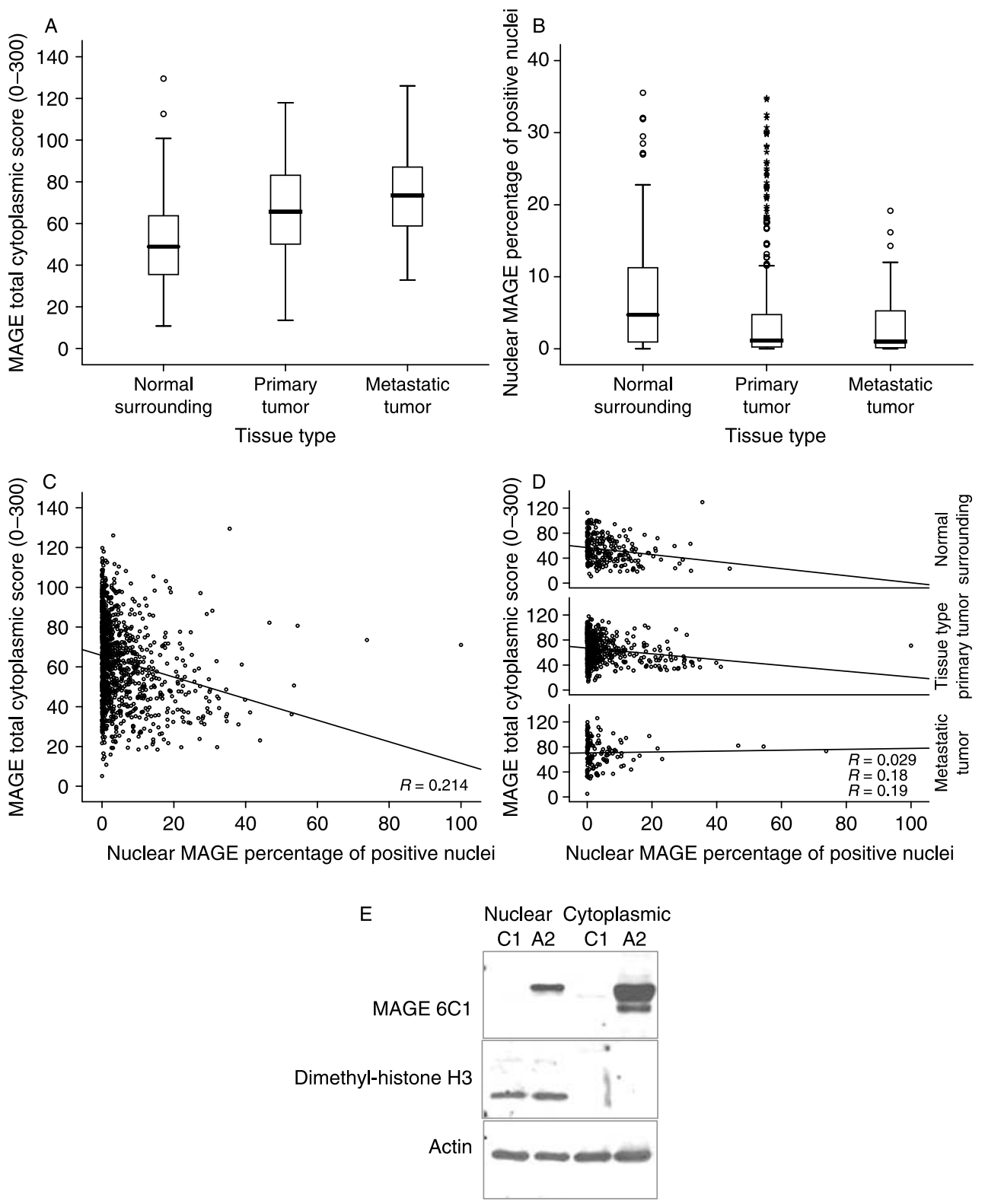

Figure 3 MAGE expression and thyroid tissue type. MAGE shows an inverse variation of cytoplasmic (A) and nuclear (B) staining with the progressively more malignant lesions. (C) This inverse correlation is confirmed with a Pearson's $R$ of $-0.214(P<0.0001)$. $(D$ and $E$ ) Western blot of both cellular fractions - nuclear and cytoplasmic - from WRO cells stably transfected with empty vector (C1) or MAGE-A3 (A2). The dimethyl-histone antibody confirms the separation of the nuclear fraction and the actin confirms an equal loading of protein. MAGE is clearly expressed in both cellular compartments with a cytoplasmic predominance. $R$, Pearson's $R$.

and its metastases. Nuclear MAGE staining was evaluated and a distribution curve was performed according to the percentage of positive nuclei per tissue core reported by the automated analyzer, as well as statistical tests of normality. These showed a nonnormal distribution with a clear cut-off below the $10 \%$ value. Nuclear staining was relatively stronger in normal tissue compared with primary tumors $(7.5 \%$ $($ S.E.M. $=0.65)$ vs $4.64 \%$ (s.E.M. $=0.37), P=0.002$ and compared with tumor metastases $4.73 \%$ (s.E.M. $=1.3$ ), $P=0.06$; Fig. 3B).

The correlation between nuclear and cytoplasmic MAGE staining in the human thyroid samples was found to be negative and significant, with a Pearson's $R$ of -0.214 and a $P<0.0001$ (Fig. 3C). When analyzed only in tumor tissues, this correlation persisted, remaining highly statistically significant (Pearson's $R=-0.18, P<0.0001$; Fig. 3D). 


\section{Fractionation experiments}

Because IHC localized MAGE to both nuclear and cytoplasmic components in human thyroid specimens, we performed fractionation experiments and western blotting. A clear reactivity for MAGE in both the nuclear and cytoplasmic compartments was identified, while the control antibody, dimethyl-histone H3, was exclusive to the nuclear fraction, supporting the immunohistochemical findings (Fig. 3E).

\section{MAGE immunoreactivity and histologic tumor type}

The histological tumor subtypes presented relevant differences of MAGE protein expression according to their immunoreactivity.

In the papillary carcinomas, the cytoplasmic stain showed a significant difference between the classic and the follicular variants (mean score 59.9 (s.E.M. $=2.4$ ) vs 67.9 (s.E.M. $=3.0$ ), $P=0.04$ ). Considering the percentage of positive nuclei, a statistically significant difference
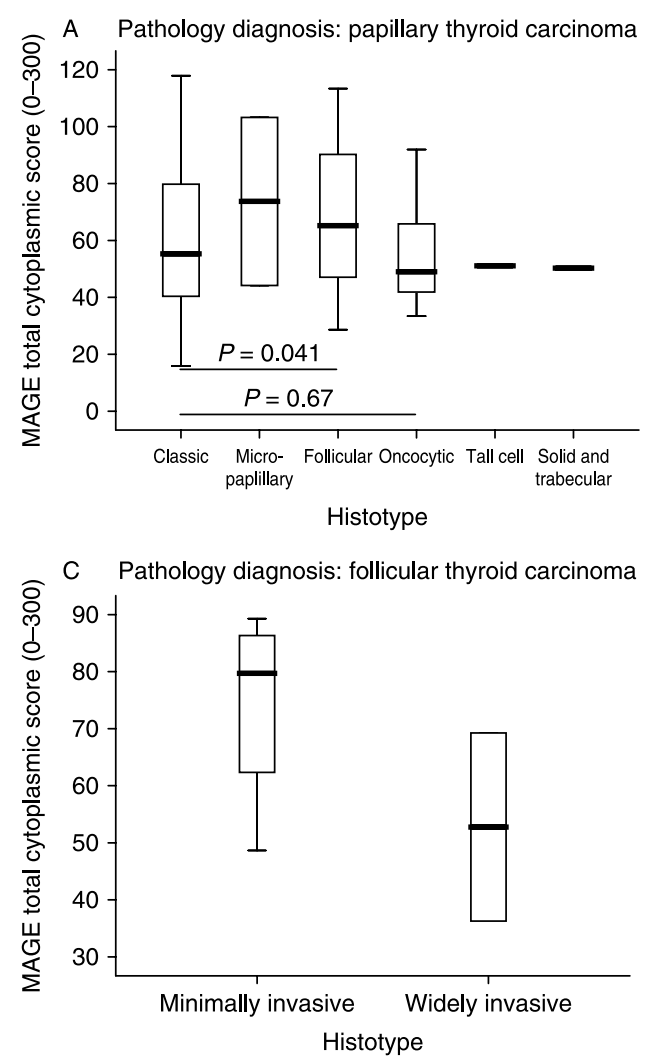

between classic and oncocytic variants was found (3.08 vs $8.4 \%, P=0.05$ ), although of poor clinical relevance. All other differences did not reach statistical significance for the rare variants, due to small numbers. In the follicular carcinomas, the cases with more than $20 \%$ nuclei positive for MAGE were widely invasive, whereas minimally invasive tumors all had $<20 \%$ positive nuclei. This difference did not reach statistical significance when comparing means $(9.6 \%$ (s.E.M. $=8.3$ ) vs $28.5 \%$ (s.E.M. $=6.2$ ), $P=0.2)$ due to sample size. When considering a cut-off value of $10 \%$ nuclei as MAGE positive, the likelihood ratio of widely invasive reached a $P$ value of 0.05 (Fig. 4).

\section{MAGE immunoreactivity and clinical variables}

Cytoplasmic MAGE staining demonstrated a positive and significant correlation with tumor size (Pearson's $R=0.084, P=0.004)$. By contrast, the percentage of cells with nuclear MAGE staining revealed no
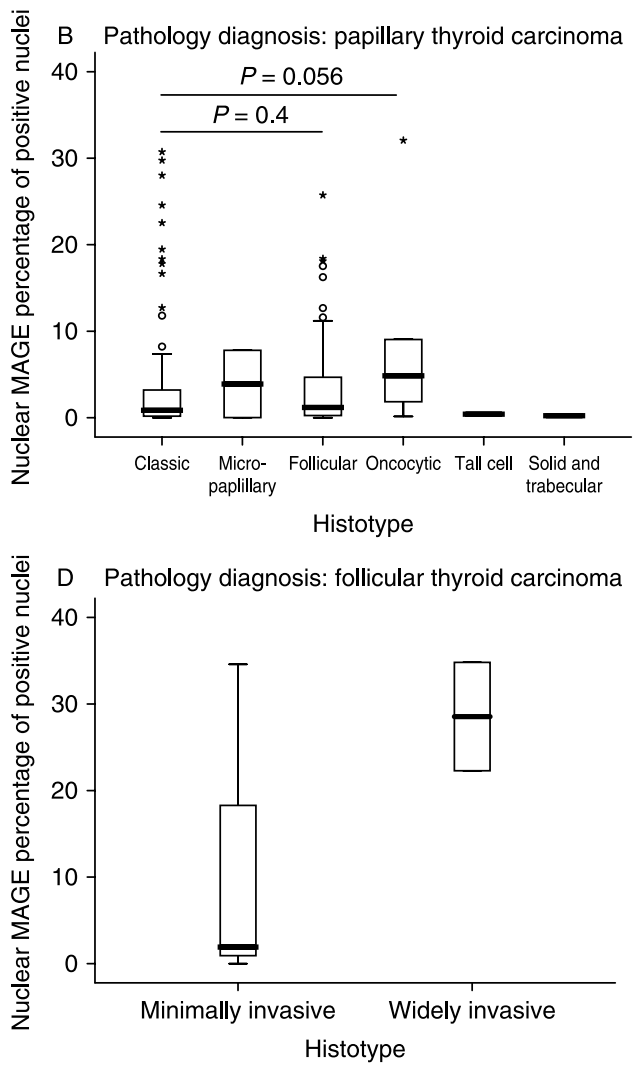

Figure 4 MAGE expression and thyroid tumor histotype. The papillary carcinomas show significant differences between the classic and follicular variants with respect to the MAGE cytoplasmic score (mean score 59.9 (s.E.M. $=2.4$ ) vs $67.9($ S.E.M. $=3.0$ ), $P=0.04$ ). Classical and oncocytic variants had clear differences in positive nuclei (3.08 vs $8.4 \%, P=0.05)$. For the other groups, no statistically significant differences were found. For the follicular cases, a clear cut-off shows that those with more than $20 \%$ nuclei positive for MAGE are widely invasive, whereas minimally invasive tumors have $<20 \%$ positive nuclei, but this result was not statistically significant (NS). 
significant association with any of the clinical variables examined.

Total cytoplasmic MAGE scores also correlated with tumor clinical behavior as classified by the AJCC/TNM system: larger tumors were associated with higher MAGE scores, independent of the presence or absence of lymph node metastases (ANOVA between groups' analysis $P<0.01$ for cytoplasmic score and percentage of positive nuclei). MAGE staining was weakly associated with the number of lymph node metastases (not significant). We also observed a positive and significant correlation between the MAGE cytoplasmic score and the number of histologically proven lymph node metastases (Pearson's $R=0.059, P=0.043$; Fig. 5). Vascular invasion also tended to correlate with MAGE score, but this was
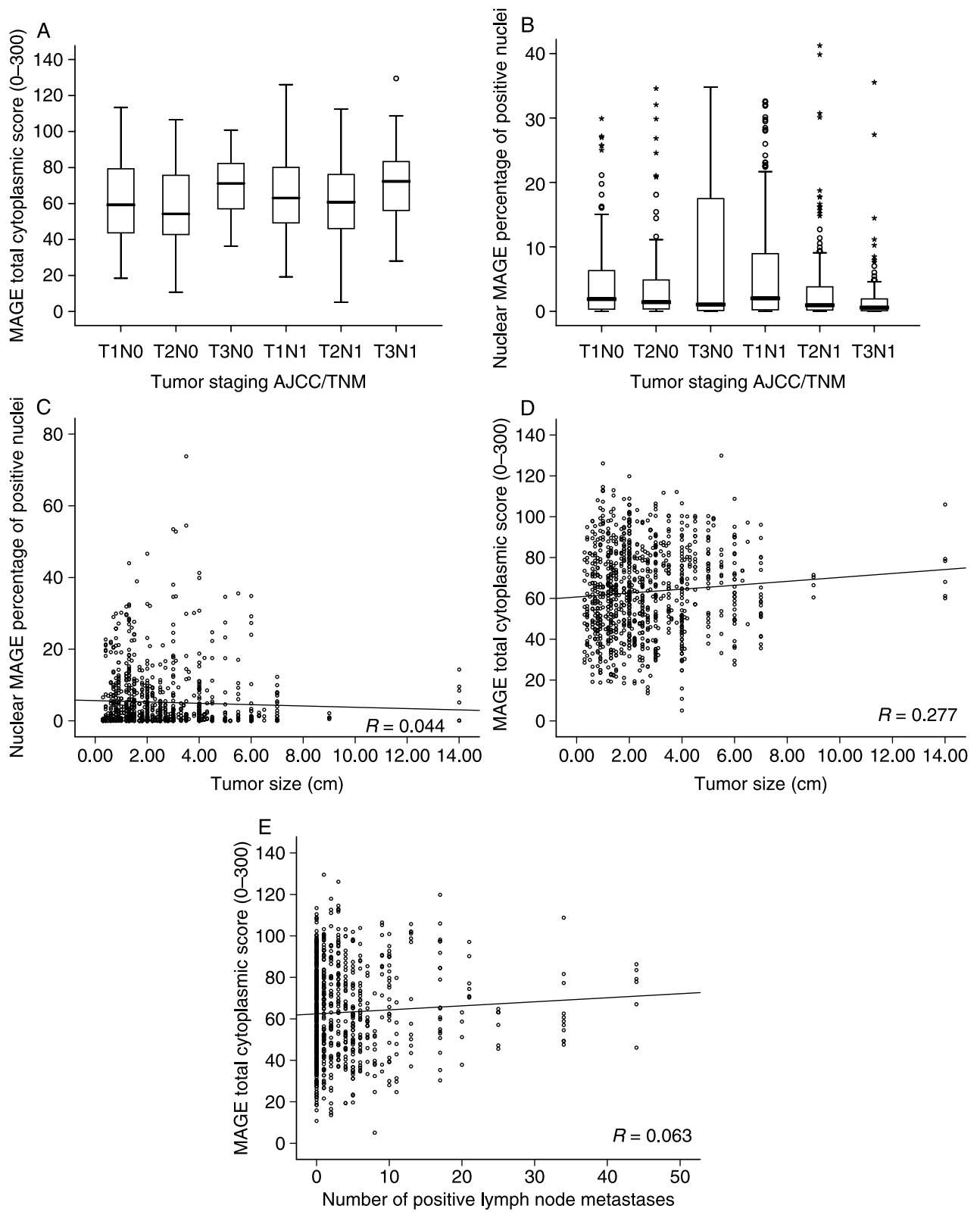

Figure 5 MAGE expression and thyroid tumor stage. (A) The cytoplasmic staining, particularly the strong intensity, showed a consistent increase - although not statistically significant - in the stages of larger size. (B) MAGE showed a higher percentage of positive nuclei in the group of larger size, independently of the presence of lymph node metastases. (C and D) MAGE expression according to tumor size: the percentage of positive nuclei is not correlated to size, but the cytoplasmic stain has a positive and significant correlation (Pearson's $R=0.084, P=0.004$ ). (E) When correlated to the number of lymph node metastases found during surgery, the MAGE cytoplasmic score showed a positive and significant association (Pearson's $R=0.059, P=0.043$ ). $R$, Pearson's $R$. 

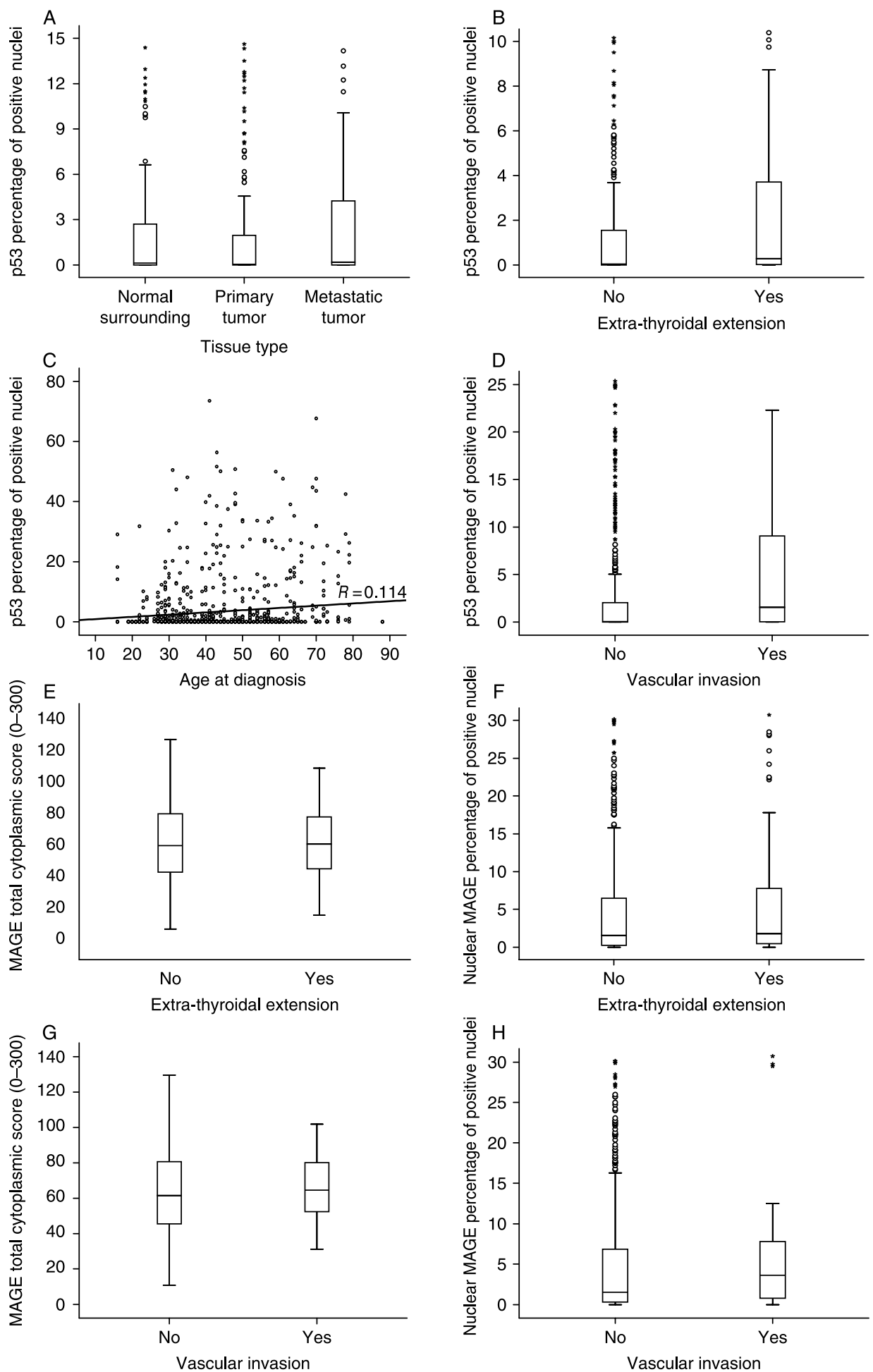

Figure 6 MAGE and p53 expressions and tumor behavior. (A) Although there seems to be a higher percentage of p53-positive nuclei in metastatic carcinomas compared with primary tumors and normal tissue, there is no statistical significance for the differences between these tissue types. (B) There is a clear association between the percentage of p53-positive nuclei and extra-thyroidal extension $(P=0.021)$. (C) The number of p53-positive nuclei was also associated with a positive and significant correlation with age at diagnosis (Pearson's $R=0.12, P=0.002$ ). (D) Although there is a suggestion of correlation between p53 nuclear staining and vascular invasion, this is not statistically significant $(P=\mathrm{NS})$. MAGE staining did not correlate with extra-thyroidal extension (E and $\mathrm{F})$ or vascular invasion $(\mathrm{G}$ and $\mathrm{H})$, whether examining cytoplasmic $(\mathrm{E}$ and $\mathrm{G})$ or nuclear $(\mathrm{F}$ and $\mathrm{H})$ positivity. $R$, Pearson's $R$. 
not statistically significant due to the small number of cases with unequivocal vascular invasion (63.1 $($ s.E.M. $=0.9)$ vs 65.8 (s.E.M. $=3.02), P=0.6$ for the cytoplasmic score).

\section{p53 immunoreactivity}

p53 showed clear nuclear staining in human thyroid specimens. In particular, we noted a higher percentage of nuclear reactivity in tumor metastases compared with primary tumors and normal tissue, although this did not reach statistical significance. A higher percentage of p53-reactive nuclei was found in cases with extra-thyroidal extension $(5.97 \%$ (s.E.M. $=0.86)$ compared with cases without extra-thyroidal extension $(3.70 \%$ (s.E.M. $=0.53), P=0.021)$. Similarly, higher p53 nuclear reactivity was noted in cases with vascular invasion compared with those that did not display this feature, although this difference did not reach statistical significance $(5.73 \%$ (s.E.M. $=2.50)$ vs $4.51 \%$ (s.E.M. $=0.56)$ ). We also noted a positive and significant correlation between the percentage of p53-positive nuclei with the age at diagnosis (Pearson's $R=0.12, P=0.002$; Fig. 6).

\section{MAGE and p53 expressions}

Given the ability of MAGE to downregulate p53 (Kondo et al. 2007b, Zhu et al. 2008), we also examined the relationship between MAGE and p53. We found a statistically significant negative correlation between MAGE expression in the cytoplasm and the percentage of p53-positive nuclei (Pearson's $R=-0.124, P<0.0001$ ). This correlation was not present when compared with the percentage of MAGE-positive nuclei. We then explored this
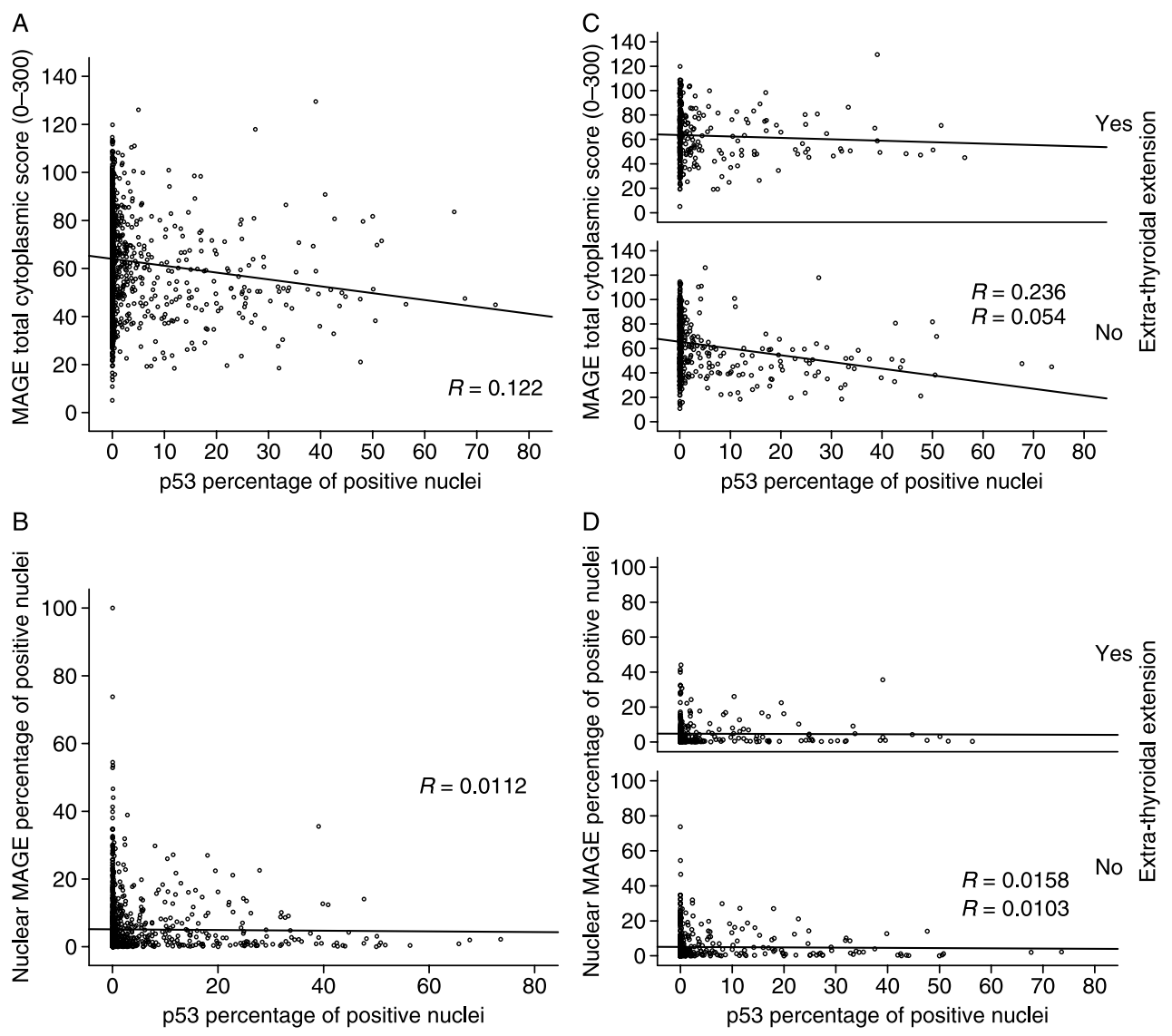

Figure 7 Correlation between MAGE and p53 staining: (A) cytoplasmic and (B) nuclear and percentage of positive nuclei for p53 for each core show a clear negative correlation (Pearson's $R=-0.124, P<0.0001$ ) between MAGE cytoplasmic staining and the percentage of p53-positive nuclei. By contrast, the correlation between the percentage of MAGE-positive nuclei and p53 is not significant (Pearson's $R=-0.016, P=0.353$ ). (C) The MAGE cytoplasmic score showed a negative correlation with p53 staining. This inverse relationship was more pronounced in cases with no extra-thyroidal extension compared with those displaying this feature $(R=-0.055(P=0.1)$ vs $R=-0.23(P=0.0001))$. (D) There was no correlation between nuclear MAGE and p53 staining. $R$, Pearson's $R$. 
correlation across the different clinical variables. In particular, we found a significant difference in the correlation between tumors with and without extra-thyroidal extension: the inverse relationship was more pronounced in cases with no extrathyroidal extension compared with those displaying this feature $(R=-0.055(P=0.1)$ vs $R=-0.23$ $(P=0.0001)$; Fig. 7).

\section{Discussion}

Although some members of the MAGE family are normally expressed in the testis and placenta, members of the A subfamily are expressed mainly in neoplastic tissues. Previous immunohistochemical studies have identified MAGE-A3 as a candidate mediator in thyroid carcinogenesis (Milkovic et al. 2006). Although this work was preliminary in terms of sample size and information regarding clinical behavior, it provided the first tissue-based evidence of the possible participation of a member of the MAGE family in thyroid cancer. Functional studies from our group have described an epigenetically controlled interaction between MAGE-A3 and FGFR2, participating in the development of thyroid cancer (Kondo et al. 2007b). Furthermore, we identified MAGE-A3 as a candidate mediator of fibronectin-mediated thyroid cancer progression (Liu et al. 2008).

The present work attempts to place these findings in a clinical context, facing the usual difficulties of thyroid cancer prognosis studies: sample size and time of follow-up. To overcome these challenges, we used high-throughput techniques of TMAs and automated analyses coupled with advanced software image analysis (Vrolijk et al. 2003). These instruments permitted the collection of large libraries of pathology material across several years, the selection of the more representative areas of tumor to be analyzed, and a refined and objective scoring system (Stromberg et al. 2007).

Our findings in human tissues show that MAGE proteins are overexpressed in primary and metastatic thyroid tumors compared with their surrounding unaffected 'normal' tissues. The differences among histotypes support their participation in different steps of thyroid oncogenesis. Perhaps most relevant was our finding of a significant correlation between MAGE cytoplasmic score and tumor size, as well as the number of lymph node metastases. This finding is consistent with our previous mouse xenograft studies, where the ectopic expression of MAGE in thyroid cancer cell lines xenografted into immunodeficient mice induced more numerous and larger lung metastases than control cells lacking MAGE (Liu et al. 2008).

p53 is a well-characterized transcription factor that participates in growth control mechanisms such as cell cycle arrest, apoptosis, senescence, and DNA repair. Loss of p53 results in deregulated cell growth, escape from apoptosis, and diminished capacity for DNA repair. Loss of p53 function is typically the result of an intragenic mutation (Bourdon et al. 2005, Toledo \& Wahl 2006, Bourdon 2007). Intragenic p53 mutations have been long recognized to be infrequent in primary human thyroid carcinomas (Ito et al. 1992); they are associated with loss of differentiation and with the most aggressive tumors (Donghi et al. 1993, Fagin et al. 1993). Nevertheless, altered p53 immunoreactivity that may not reflect mutation has been described in several studies (Hosal et al. 1997). The current study using a relatively large-sample size again demonstrated variable p53 immunoreactivity in thyroid carcinomas. Furthermore, given the recognized ability of MAGE to interact with epigenetic controllers such as histone deacetylase to downregulate the p53 promoter (Yang et al. 2007), we were provided with an opportunity to examine the relationship between MAGE expression and diminished p53 expression. Indeed, supported by such transfection studies, we identified a negative correlation between MAGE and p53 (Liu et al. 2008). Interestingly, this inverse relationship was even more pronounced in tumors that did not display extra-thyroidal extension, suggesting uncoupling of the MAGE-p53 relationship with disease progression. Nevertheless, the MAGE-associated loss of p53 is now supported in differentiated primary human specimens.

It is anticipated that the current findings will facilitate the application of MAGE as an additional tumor marker in thyroid specimens with equivocal morphologic features. It may also provide a better understanding of the potential mediators involved in disruption of p53 action in papillary thyroid carcinomas.

The lack of highly specific antibodies to identify MAGE family members is a limiting factor. We obtained two antibodies, but were unable to prove the specificity of one of these for our studies. Although the $6 \mathrm{C} 1$ antibody is not specific for one single member of the family of MAGE proteins, we were able to prove its ability to localize fractionated MAGE in human MAGE-A3-transfected cells. Furthermore, this antibody effectively displayed a strong association of MAGE immunoreactivity with tissue type and tumor behavior. Future studies using 
yet-to-be-developed, more specific antibodies for the different members of the A subgroup of MAGE proteins might validate more dramatic differences between risk groups. This would allow the addition of MAGE to the arsenal of possible tumor markers applied in the clinical setting to refine diagnostic procedures such as fine-needle biopsies, or for prognostic purposes, helping the treating physician to decide between an aggressive and a conservative course of therapy.

\section{Declaration of interest}

The authors declare that there is no conflict of interest that could be perceived as prejudicing the impartiality of the research reported.

\section{Funding}

This work was supported in part by the Canadian Institutes of Health Research/CBCRA (MOP-86493), the Toronto Medical Laboratories, and the Rita Banach Thyroid Cancer Research Fund.

\section{Acknowledgements}

The authors acknowledge the contribution of material from Dr Lourdes Cabrera and the technical assistance of Kelvin So and Heather Begley.

\section{References}

Asa SL 2005 The role of immunohistochemical markers in the diagnosis of follicular-patterned lesions of the thyroid. Endocrine Pathology 16 295-309.

St Bernard R, Zheng L, Liu W, Winer D, Asa SL \& Ezzat S 2005 Fibroblast growth factor receptors as molecular targets in thyroid carcinoma. Endocrinology 146 $1145-1153$.

Bourdon JC 2007 p53 Family isoforms. Current Pharmaceutical Biotechnology 8 332-336.

Bourdon JC, Fernandes K, Murray-Zmijewski F, Liu G, Diot A, Xirodimas DP, Saville MK \& Lane DP 2005 p53 isoforms can regulate p53 transcriptional activity. Genes and Development 19 2122-2137.

Chitale DA, Jungbluth AA, Marshall DS, Leitao MM, Hedvat CV, Kolb D, Spagnoli GC, Iversen K \& Soslow RA 2005 Expression of cancer-testis antigens in endometrial carcinomas using a tissue microarray. Modern Pathology 18 119-126.

Donghi R, Longoni A, Pilotti S, Michieli P, Della Porta G \& Pierotti MA 1993 Gene p53 mutations are restricted to poorly differentiated and undifferentiated carcinomas of the thyroid gland. Journal of Clinical Investigation 91 1753-1760.
Fagin JA, Matsuo K, Karmakar A, Chen DL, Tang SH \& Koeffler HP 1993 High prevalence of mutations of the p53 gene in poorly differentiated human thyroid carcinomas. Journal of Clinical Investigation 91 179-184.

Fischer C, Gudat F, Stulz P, Noppen C, Schaefer C, Zajac P, Trutmann M, Kocher T, Zuber M, Harder F et al. 1997 High expression of MAGE-3 protein in squamous-cell lung carcinoma. International Journal of Cancer $\mathbf{7 1}$ $1119-1121$.

Hosal SA, Apel RL, Freeman JL, Azadian A, Rosen IB, LiVolsi VA \& Asa SL 1997 Immunohistochemical localization of p53 in human thyroid neoplasms: correlation with biological behavior. Endocrine Pathology 8 21-28.

Hudolin T, Juretic A, Pasini J, Tomas D, Spagnoli GC, Heberer M, Dimanovski J \& Kruslin B 2006 Immunohistochemical expression of tumor antigens MAGE-A1, MAGE-A3/4, and NY-ESO-1 in squamous cell carcinoma of the penis. Urology 68 205-207.

Ito T, Seyama T, Mizuno T, Tsuyama N, Hayashi T, Hayashi Y, Dohi K, Nakamura N \& Akiyama M 1992 Unique association of p53 mutations with undifferentiated but not with differentiated carcinomas of the thyroid gland. Cancer Research 52 1369-1371.

Jang SJ, Soria JC, Wang L, Hassan KA, Morice RC, Walsh GL, Hong WK \& Mao L 2001 Activation of melanoma antigen tumor antigens occurs early in lung carcinogenesis. Cancer Research 61 7959-7963.

Kobayashi Y, Higashi T, Nouso K, Nakatsukasa H, Ishizaki M, Kaneyoshi T, Toshikuni N, Kariyama K, Nakayama E \& Tsuji T 2000 Expression of MAGE, GAGE and BAGE genes in human liver diseases: utility as molecular markers for hepatocellular carcinoma. Journal of Hepatology 32 612-617.

Kondo T, Ezzat S \& Asa SL 2006 Pathogenetic mechanisms in thyroid follicular-cell neoplasia. Nature Reviews. Cancer 6 292-306.

Kondo T, Zheng L, Liu W, Kurebayashi J, Asa SL \& Ezzat S $2007 a$ Epigenetically controlled fibroblast growth factor receptor 2 signaling imposes on the RAS/BRAF/ mitogen-activated protein kinase pathway to modulate thyroid cancer progression. Cancer Research $\mathbf{6 7}$ 5461-5470.

Kondo T, Zhu X, Asa SL \& Ezzat S 2007b The cancer/testis antigen melanoma-associated antigen-A3/A6 is a novel target of fibroblast growth factor receptor 2-IIIb through histone $\mathrm{H} 3$ modifications in thyroid cancer. Clinical Cancer Research 13 4713-4720.

Liu W, Wei W, Winer D, Bamberger AM, Bamberger C, Wagener C, Ezzat S \& Asa SL 2007 CEACAM1 impedes thyroid cancer growth but promotes invasiveness: a putative mechanism for early metastases. Oncogene $\mathbf{2 6}$ 2747-2758.

Liu W, Cheng S, Asa SL \& Ezzat S 2008 The melanomaassociated antigen A3 mediates fibronectin-controlled cancer progression and metastasis. Cancer Research 68 8104-8112. 
Milkovic M, Sarcevic B \& Glavan E 2006 Expression of MAGE tumor-associated antigen in thyroid carcinomas. Endocrine Pathology 17 45-52.

Monte M, Simonatto M, Peche LY, Bublik DR, Gobessi S, Pierotti MA, Rodolfo M \& Schneider C 2006 MAGE-A tumor antigens target p53 transactivation function through histone deacetylase recruitment and confer resistance to chemotherapeutic agents. PNAS 103 11160-11165.

Picard V, Bergeron A, Larue H \& Fradet Y 2007 MAGE-A9 mRNA and protein expression in bladder cancer. International Journal of Cancer 120 2170-2177.

Stromberg S, Björklund MG, Asplund C, Skollermo A, Persson A, Wester K, Kampf C, Nilsson P, Andersson AC, Uhlen M et al. 2007 A high-throughput strategy for protein profiling in cell microarrays using automated image analysis. Proteomics 7 2142-2150.

Toledo F \& Wahl GM 2006 Regulating the p53 pathway: in vitro hypotheses, in vivo veritas. Nature Reviews. Cancer 6 909-923.
Tsuneyama K, Sasaki M, Shimonishi T \& Nakanuma Y 2004 Expression of MAGE-A3 in intrahepatic cholangiocarcinoma and its precursor lesions. Pathology International 54 181-186.

Vrolijk H, Sloos W, Mesker W, Franken P, Fodde R, Morreau H \& Tanke H 2003 Automated acquisition of stained tissue microarrays for high-throughput evaluation of molecular targets. Journal of Molecular Diagnostics 5 160-167.

Yang B, O'Herrin SM, Wu J, Reagan-Shaw S, Ma Y, Bhat KM, Gravekamp C, Setaluri V, Peters N, Hoffmann FM et al. 2007 MAGE-A, mMage-b, and MAGE-C proteins form complexes with KAP1 and suppress p53-dependent apoptosis in MAGE-positive cell lines. Cancer Research 67 9954-9962.

Zhu X, Asa SL \& Ezzat S 2008 Fibroblast growth factor 2 and estrogen control the balance of histone 3 modifications targeting MAGE-A3 in pituitary neoplasia. Clinical Cancer Research 14 1984-1996. 